\title{
PRELIMINAR PRIORITIZATION OF CLINICAL VARIABLES OF THE RESPIRATORY SYSTEM OF NEONATAL PATIENTS USING THE ANALYTICAL HIERARCHY PROCESS.
}

\begin{abstract}
In this paper, we applied a Multicriteria Decisions Method (MCDM) to identify which respiratory clinical variables require urgent evaluation when a neonatal patient is being evaluated. To do so, the Analytical Hierarchy Process (AHP) was used to perform the prioritization on Silvermann-Andersen Test.
\end{abstract}

Keywords: Silverman-Andersen Score, Priorization, AHP.

\section{Introduction}

The Silverman-Andersen (SA) score is a clinical procedure commonly used for evaluating the respiratory effort of neonatal patients. This allows determining the required clinical procedures. The score is composed by the non-weighted sum of five variables (Upper Chest Retractions, Intercostal Retractions, Xiphoid Retractions, nares dilatation and expiration grunt), which implies that all the variables are equally important. However, in medical practice this is not the case since the importance of a given variable depends on other factors, such as: temperature, gestational age, congenital disease, etc.

In this work, we used the Analytical Hierarchy Process (AHP) to prioritize the SA variables when they are influenced by temperature and gestational age.

\section{Silverman-Andersen Score}

Dr.William Silverman and Dr. Dorothy Andersen created the test in 1955. The clinical trial consists of the doctor or specialist giving a score between zero and two for each variable. The values shows the neonatal behavior for each variable in three levels: absent (0) moderated (1) and severe (2). Then, the sum of the scores is calculated in order to provide an idea about the patient respiratory effort. A Silverman-Andersen score between 0-3 represents a minor effort, 4-6 a moderated effort and 7-10 shows a great respiratory effort.

\section{Research Design/Methodology}

\subsection{Problem Formulation.}

In addition to the SA variables, there are other variables which affect the diagnosis process. For this reason, we used AHP to adapt SA to a broader range according to the clinical practice and to prioritize the given variables.

\subsection{Alternatives and selection of criteria}

First, a meeting with two Pediatrics and Neonatology experts of the National University of Colombia was held in order to define the main clinical variables that have influence in the respiratory health state of neonatal patients. The group of experts selected the five variables of SA and the respiratory frequency as alternatives of our study. In addition, the temperature, gestational age and the order of apparition of the variables were chosen as important criteria. All criterion were selected based on the experience of professionals. 3.3. Data collecting 
The data was collected by applying a survey of 97 pairwise questions. A group of three Pediatricians and neonatologists of National University of Colombia answered these surveys. The opinion of experts were considered with the same weight.

3.4 Inconsistencies found

During the matrix analysis, several inconsistencies were found. In most of the cases, the experts were choosing "very important" for all the comparisons. Hence, an explanation about the inconsistencies in their judgment was given to them and the surveys were applied again. In addition, we added one expert more.

\section{Results}

The results obtained are shown in the figure 1 and 2 . It can be seen that the physical (Xiphoid retraction) and sonorous alterations (expiration grunt) have a higher priority than the others. This can be explained since this variables help to determine quickly the state of the respiratory system of the neonate.

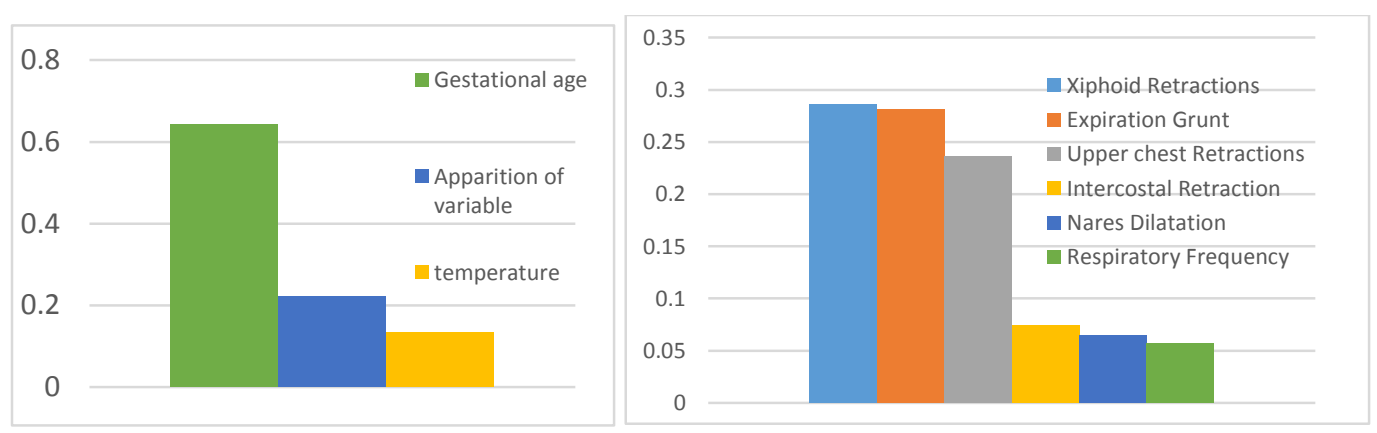

Figure 1. Weight of the criteria of the first level

Fig. 2. Prioritization of the clinical variables of respiratory system of neonatal patients.

\section{Conclusions}

We propose a Priorization for the variables of the respiratory system by considering temperature, gestational age and the order of apparition of the variables. For future studies, we can include more criteria that will allow us to reduce the sensibility between variables and criteria and add more experts to enforce the results.

This work shows that the priority for each variable in the Silverman-Andersen scale is not evenly considered in the medical exercise. This is because there are certain symptoms that indicate conditions that are more severe and therefore, it is erroneous to consider all the variables with equal importance.

\section{Key References}

-Saaty T.L. (1980). The analytical Hierarchy process. RWS Publications. Pittsburgh.

-Silverman W., Andersen D. (1955) Controlled clinical trial of effects of alevaire mist on prematury infants. JAMA 1955; 157:1093

-Sharma P.,Mckay K., Rosenkrantz T.,Hussain N.(2004).Comparisons of mortality and pre-discharge respiratory outcomes in small-for-gestational-age and appropriate-forgestational-age premature infants. BMC Pediatrics 2004 Jun 08; Vol. 4, pp. 9.

-Forsythe E., Allen P. (2013) Health Risks Associated with Late- Preterm Infants: Implications for Newborn Primary Care. Pediatric Nursing. Jul/Aug2013, Vol. 39 Issue 4, p197-201. 5p. 1 Chart. 